\title{
Mechanism and therapeutic effects of Saccharomyces boulardii on experimental colitis in mice
}

\author{
HUAN ZHOU, HUI-JING ZHANG, LIN GUAN, YI-NING ZHANG, \\ YUE LI and MING-JUN SUN
}

\author{
Department of Gastroenterology and Endoscopy, The First Affiliated Hospital of
}

China Medical University, Shenyang, Liaoning 11000, P.R. China

Received January 10, 2018; Accepted October 10, 2018

DOI: $10.3892 / \mathrm{mmr} .2018 .9612$

\begin{abstract}
Inflammatory bowel disease (IBD) is a type of chronic inflammatory disturbance that affects a number of individuals worldwide; the precise mechanism is unclear and treatment is frequently insufficient to maintain patients in remission. Saccharomyces boulardii is a thermophilic, non-pathogenic yeast that may be administered for prophylaxis and treatment of a variety of diarrheal diseases. Recent clinical studies have demonstrated that it may have a role in IBD; however, the mechanism of action is unclear. The hypoxia-inducible factors (HIFs) are ubiquitously expressed regulators of cellular adaptation to hypoxia and are central to the adaptive and inflammatory responses of cells of the intestinal mucosa in patients with IBD. The present study aimed to investigate the effects of $S$. boulardii on dextran sulfate sodium (DSS)-induced colitis in mice and the effects of $S$. boulardii on HIFs. Mice were divided into five groups $(\mathrm{n}=10$ mice/group): i) Control; ii) DSS; iii) S. boulardii (Sb) + DSS; iv) normal saline (NS) + DSS; and v) $S b$. For 14 consecutive days, mice from the $S b+\mathrm{DSS}$ and $S b$ groups were given $S$. boulardii suspension in saline $(150 \mathrm{mg} / \mathrm{kg} /$ day; final volume $0.2 \mathrm{ml})$ by oral gavage. The NS+DSS group received the same volume of NS by gavage. The Control mice received water only. From day 8 to day $14,3.5 \%$ DSS was added to the drinking water of
\end{abstract}

Correspondence to: Professor Ming-Jun Sun, Department of Gastroenterology and Endoscopy, The First Affiliated Hospital of China Medical University, 155 Nanjing North Street, Heping, Shenyang, Liaoning 11000, P.R. China

E-mail: sunmingjun2175@hotmail.com

Abbreviations: DSS, dextran sulfate sodium; EMT, epithelialmesenchymal transition; HIF hypoxia-inducible factor; IBD, inflammatory bowel disease; VEGF, vascular endothelial growth factor

Key words: inflammatory bowel disease, dextran sulfate sodiuminduced colitis, Saccharomyces boulardii, hypoxia-inducible factor, epithelial-mesenchymal transition the DSS, $S b+\mathrm{DSS}$ and NS+DSS groups to induce acute colitis. Body weight decreased and disease activity index and histological score increased in mice with DSS-induced colitis. Oral administration of $S$. boulardii reduced DSS-induced weight loss, ameliorated the histological damage and protected the colon barrier in mice with DSS-induced colitis. The expression of HIF-1 $\alpha$ and HIF- $2 \alpha$ in colon tissues was measured by reverse transcription-quantitative polymerase chain reaction, immunoblotting and immunohistochemistry. The increase in HIFs in the colon induced by DSS was significantly inhibited by $S$. boulardii treatment. The expression levels of several epithelial-mesenchymal transition (EMT) markers and of vascular endothelial growth factor (VEGF) that are regulated by HIFs were measured. $S$. boulardii reduced EMT and decreased expression of VEGF that was induced by DSS treatment. These results indicated that treatment with $S$. boulardii ameliorated DSS-induced colitis, partly through downregulation of HIF- $1 \alpha$ and HIF- $2 \alpha$.

\section{Introduction}

Inflammatory bowel disease (IBD) is a serious health problem worldwide, and the incidence of IBD has increased annually. The highest annual incidence of UC was from 6.3 to 24.3 per 100,000 person-years. The highest annual incidence of CD was from 5.0 to 20.2 per 100,000 person-years (1). It seriously affects quality of life and has a major social and economic impact. The annual cost of treatment puts a heavy burden on patients and families, so it is not only a medical problem but also a social issue. The exact etiology and pathogenesis of IBD is not completely known. However, hypoxia serves an important function in the inflammatory and injury response (2). Colonic mucosal hypoxia may occur in patients with IBD. When the availability of oxygen is a limiting factor, tissues become hypoxic, which results in the activation of adaptive pathways to enable the survival of hypoxic episodes. The primary pathway activated is the hypoxia inducible factor (HIF) pathway, which is central to the adaptive and inflammatory responses of cells of the intestinal mucosa in patients with IBD (3). Experimental and clinical studies have reported that HIF-1 $\alpha$ and HIF-2 $\alpha$ are activated in IBD patients $(2,4)$. 
At present, 5-aminosalicylic acid and its analogs, corticosteroids or alternative immunomodulatory drugs are used for the treatment of active IBD. However, maintenance of remission is limited, and the irreversible and the severe adverse drug effects should not be ignored (5,6). Given the limitations of current standards of practice in prevention and treatment of IBD, it is essential to investigate alternative strategies with high efficacy, but with fewer toxic adverse effects.

The role of intestinal flora in the pathogenesis of IBD is notable. Molecular and genome-wide studies have identified distinct alterations in the gut microbiota of IBD and have elucidated the importance of dysbiosis of the gut microbiota in the etiopathogenesis of IBD $(7,8)$. In the human body, probiotics have a nutritional value and serve important roles in protection of the intestinal mucosa, regenerating and maintaining the integrity and stability of the environment. Therefore, the application of probiotics in the treatment of IBD has become a research focus in recent years.

The non-pathogenic yeast Saccharomyces boulardii has been demonstrated to be effective in the prophylaxis and the treatment of a variety of diarrheal diseases (9). It is suggested that this probiotic yeast has beneficial properties, including improving the gut immune response and the intestinal barrier (10,11). Previous clinical studies have indicated that $S$. boulardii may also be effective in IBD $(12,13)$. However, the mechanisms underlying the protective actions of $S$. boulardii are not well understood; in addition, the relationship between S. boulardii and HIF is unknown. The aim of the present study was to examine the effects of $S$. boulardii treatment in a mouse model of dextran sulfate sodium (DSS)-induced colitis and to investigate the underlying mechanisms through the examination of the expression levels of HIF- $1 \alpha$ and HIF- $2 \alpha$ in mice with DSS-induced colitis.

\section{Materials and methods}

Animals and experimental design. A total of 50 male BALB/c mice (age, 6-8 weeks) were purchased from the Center of Experimental Animals of China Medical University (Shenyang, China). The mice were housed 5 per cage in a clean animal room under standard conditions of temperature $\left(25 \pm 2^{\circ} \mathrm{C}\right)$ and humidity (50-60\%) on a 12-h light/dark cycle and were fed with standard laboratory chow and water ad libitum. All animal experiments were performed in accordance with the Animals Scientific Procedures Act (1986) and were approved by the Ethics Review Committee for Animal Experimentation of the China Medical University (Liaoning, China).

Following a 7-day acclimation period, the mice were randomly divided into five groups of ( $\mathrm{n}=10 \mathrm{mice} / \mathrm{group}$ ): i) Control; ii) DSS only; iii) S. boulardii $(\mathrm{Sb})+\mathrm{DSS}$; iv) normal saline (NS) + DSS; and v) $S b$ only. For 14 consecutive days, mice in the $S b+\mathrm{DSS}$ and $S b$-only groups were given a suspension of $S$. boulardii in saline $(150 \mathrm{mg} / \mathrm{kg} /$ day; final volume $0.2 \mathrm{ml}$ ) by oral gavage. Mice in the NS+DSS group received the same volume of NS by gavage. The Control mice received water only. From day 8 , mice in the DSS, $S b+\mathrm{DSS}$ and NS+DSS groups received 3.5\% DSS (MP Biomedicals, LLC, Santa Ana, CA, USA) added to the drinking water to induce acute colitis. Mice were weighed daily and evaluated for clinical signs of colitis. Following 14 days of treatments, all mice were sacrificed and the colons were excised, measured and sectioned for further analysis.

Clinical evaluation of colitis. To determine the general condition of the mice, the disease activity index (DAI) was determined, as described previously (14) (Table I). Mice were weighed daily and inspected for stool consistency, presence of blood in stool and bleeding. Alteration of body weight was determined.

Histopathological analysis. Following 14 days of treatments, mice were sacrificed and excised colons were fixed in $4 \%$ paraformaldehyde for $24 \mathrm{~h}$ at $4{ }^{\circ} \mathrm{C}$, dehydrated in a graded ethanol series ( $75 \%$ for $2 \mathrm{~h}, 85 \%$ for $2 \mathrm{~h}, 95 \%$ overnight, absolute ethanol for $1 \mathrm{~h} \times 2$ times, xylene for $10 \mathrm{~min} \times 2$ times) and embedded in paraffin. Subsequently, paraffin-embedded samples were sectioned $(4 \mu \mathrm{m})$ and stained with hematoxylin and eosin $(\mathrm{H} \& \mathrm{E})$ at room temperature $(0.2 \%$ hematoxylin staining for $5 \mathrm{~min}$ and $0.5 \%$ eosin staining for $5 \mathrm{~min}$ ). Inflammation was graded from 0 to 4 , as described previously (15). To evaluate the severity of inflammation, 9 randomly selected fields (magnification, $\mathrm{x} 200$ ) were inspected in each section with an inverted fluorescence microscope by two pathologists blinded to the experimental groups. Histological damage was assessed by inflammation, lesion depth, crypt destruction score and lesion range score. The mean score was taken as a histological score for colonic tissue injury.

RNA isolation and reverse transcription-quantitative polymerase chain reaction ( $R T-q P C R)$. Total RNA was extracted using the TRIzol ${ }^{\circledR}$ reagent (Invitrogen; Thermo Fisher Scientific, Inc., Waltham, MA, USA) from mouse tissue segments ( $\sim 5 \mathrm{~mm}$ long) and was reverse transcribed into cDNA with PrimeScript ${ }^{\mathrm{TM}}$ RT reagent kit with gDNA Eraser (Takara Bio, Inc., Otsu, Japan). qPCR was performed using the SYBR PremixEx Taq ${ }^{\mathrm{TM}}$ II (Takara Bio, Inc., Otsu, Japan) on a Real-Time PCR System (GeneAmp PCR System 9600, PerkinElmer, Inc., Waltham, MA, USA) under the following cycling conditions: Initial denaturation at $95^{\circ} \mathrm{C}$ for $30 \mathrm{sec}$; followed by 40 cycles of $95^{\circ} \mathrm{C}$ for $5 \mathrm{sec}$ and $60^{\circ} \mathrm{C}$ for $30 \mathrm{sec}$. Melting curve analysis was performed to ensure amplification of single PCR products. $\beta$-actin was used as an endogenous control. All experiments were performed in triplicate. Reactions with no template were included as negative controls. Relative mRNA expression levels of target genes were calculated using the $2^{-\Delta \Delta C q}$ method (16). Results were expressed as a fold-change relative to the control animals. Primers were synthesized by Sangon Biotech Co., Ltd., (Shanghai, China; Table II).

Western blotting. Western blotting was used to detect protein expression levels. The appropriate volume of radioimmunoprecipitation assay buffer (Beyotime Institute of Biotechnology, Shanghai, China) and $1 \%$ phenylmethylsulfonyl fluoride was added to the colon tissues according to their size $(\sim 5 \mathrm{~mm}$ long) and the tissues were homogenized on ice, following the manufacturer's protocol. The lysates were centrifuged at $12,000 \mathrm{x} \mathrm{g}$ for $15 \mathrm{~min}$ at $4^{\circ} \mathrm{C}$, and the supernatants were collected. The protein concentrations were determined using a BCA protein concentration determination kit (Beyotime 
Table I. Disease activity index. ${ }^{\mathrm{a}}$

\begin{tabular}{lccc}
\hline Stool consistency & Bleeding & Weight loss $(\%)$ & Score \\
\hline Normal & Normal & None & 0 \\
& & $1-5$ & 1 \\
Loose stools & Occult & $5-10$ & 2 \\
Diarrhea & \multirow{2}{*}{ Gross } & $10-15$ & 3 \\
& & $>15$ & 4 \\
\hline
\end{tabular}

${ }^{\mathrm{a} D i s e a s e}$ activity index $=($ weight loss + stool consistency + bleeding $) / 3$.

Institute of Biotechnology). Equal amounts (50 $\mu \mathrm{g})$ of each protein sample were separated by SDS-PAGE (5\% concentrated gel and $10 \%$ separating gel) under denaturing conditions, followed by transfer to polyvinylidene fluoride membranes (EMD Millipore, Billerica, MA, USA). The membranes were blocked in TBS + Tween-20 buffer $(0.05 \%$ Tween-20) containing 5\% skimmed milk for $2 \mathrm{~h}$ at room temperature and subsequently incubated overnight at $4^{\circ} \mathrm{C}$ with the following primary antibodies: Mouse monoclonal anti-human occludin (1:1,000; cat. no. 611090; BD Biosciences, Franklin Lakes, NJ, USA); rabbit polyclonal anti-human claudin-1 (1:1,000; cat. no. 4933S; Cell Signaling Technology, Inc., Danvers, MA, USA); mouse monoclonal anti-human HIF-1 $\alpha$ (1:200; cat. no. ab1; Abcam, Cambridge, UK); mouse monoclonal anti-human epithelial (E)-cadherin (1:5,000; cat. no. 610404; BD Biosciences); rabbit polyclonal anti-human vimentin (1:2,000; cat. no. 5741S; Cell Signaling Technology, Inc.); rabbit polyclonal anti-human HIF-2 $\alpha$ (1:500; cat. no. ab199; Abcam); mouse monoclonal anti-human vascular endothelial growth factor (VEGF; 1:200; cat. no. sc7269; Santa Cruz Biotechnology, Inc., Dallas, TX, USA); mouse monoclonal anti-human $\beta$-actin $(1: 6,000$; cat. no. ZM0001, OriGene Technologies, Inc., Beijing, China). $\beta$-actin was used as an endogenous control. Following repeated washes with TBS-T buffer, the membranes were incubated with horseradish peroxidase-conjugated goat anti-rabbit secondary antibody (1:5,000; cat. no. ZF-0316; OriGene Technologies, Inc.) or goat anti-mouse secondary antibody (1:5,000; cat. no. ZF-0312; OriGene Technologies, Inc.) for $2 \mathrm{~h}$ at room temperature. Protein bands were visualized using the Western Lightning Chemiluminescence Reagent Plus kit (PerkinElmer, Inc.) and detected using a Bio-Imaging System (BioTek Instruments, Inc., Winooski, VT, USA). The levels of protein expression were quantified by ImageJ software version 1.8.0 (National Institutes of Health, Bethesda, MD, USA) and normalized to the endogenous control. All of the experiments were performed in triplicate.

Immunohistochemical analysis. Paraffin-embedded sections $(4 \mu \mathrm{m})$ were heated for $45 \mathrm{~min}$ at $60^{\circ} \mathrm{C}$ and then deparaffinized in xylene, and rehydrated in a graded alcohol series. Antigen retrieval was performed by boiling the sections in $0.01 \mathrm{M}$ citrate buffer for $20 \mathrm{~min}$ and pretreated with $3 \%$ hydrogen peroxide in PBS buffer to quench the endogenous peroxidase. Following blocking with $10 \%$ goat serum (Beyotime Institute of Biotechnology) at $37^{\circ} \mathrm{C}$ for $30 \mathrm{~min}$,
Table II. Primer sequences used for reverse transcription-quantitative polymerase chain reaction.

\begin{tabular}{ll}
\hline Gene & \multicolumn{1}{c}{ Primer sequence $\left(5^{\prime} \rightarrow 3^{\prime}\right)$} \\
\hline Occludin & F: TACGGAGGTGGCTATGGAG \\
R: AGGAAGCGATGAAGCAGAAG \\
Flaudin-1 & R: TTCCGATAACCATCATCAACAG \\
$\beta$-actin & F: GAGACCTTCAACACCCCAGC \\
& R: ATGTCACGCACGATTTCCC \\
HIF-1 $\alpha$ & F: GCGATGACACAGAAACTGAAGA \\
& R: TTCCGATGAAGGTAAAGGAGAC \\
HIF-2 $\alpha$ & F: AGCAGTTGGAAAGCAGGAAG \\
& R: GCCGAAATGTAATGGTGGAT
\end{tabular}

E, epithelial; F, forward; HIF, hypoxia inducible factor; R, reverse.

sections were incubated with primary antibodies (1:100) at $4^{\circ} \mathrm{C}$ overnight, followed by washing three times with PBS. The primary antibodies were: Mouse monoclonal anti-human occludin (cat. no. 611090; BD Biosciences); rabbit polyclonal anti-human claudin-1 (cat. no. 4933S; Cell Signaling Technology, Inc.); mouse monoclonal anti-human HIF-1 $\alpha$ (cat. no. ab1; Abcam); mouse monoclonal anti-human epithelial (E)-cadherin (cat. no. 610404; BD Biosciences); rabbit polyclonal anti-human vimentin (cat. no. 5741S; Cell Signaling Technology, Inc.); rabbit polyclonal anti-human HIF-2 $\alpha$ (cat. no. ab199; Abcam); mouse monoclonal anti-human vascular endothelial growth factor (VEGF; cat. no. sc7269; Santa Cruz Biotechnology, Inc.). Secondary antibodies [SignalStain ${ }^{\circledR}$ Boost IHC Detection Reagent (HRP, Rabbit), cat. no. 8114S, or SignalStain ${ }^{\circledR}$ Boost IHC Detection Reagent (HRP, Mouse) cat. no. 8125S; Cell Signaling Technology, Inc.] were added and incubated at room temperature for $1 \mathrm{~h}$. Signals were detected using the Diaminobenzidine Substrate kit (Maxim Biotech, Inc., Rockville, MD, USA). Counterstaining with $0.2 \%$ hematoxylin was performed for $2 \mathrm{~min}$ at room temperature and the sections were dehydrated in a graded ethanol series ( $75 \%$ for $2 \mathrm{~h}, 85 \%$ for $2 \mathrm{~h}, 95 \%$ overnight, absolute ethanol for $1 \mathrm{~h} \times 2$ times, xylene for $10 \min \times 2$ times). To evaluate the staining results, 9 randomly selected fields (magnification, $\mathrm{x} 200$ ) were inspected in each section using an inverted fluorescence microscope by two pathologists blinded to the experimental groups.

Statistical analysis. Data were analyzed with GraphPad Prism 6.0 software (GraphPad Software, Inc., La Jolla, CA, USA). Results are presented as the mean \pm standard deviation. Differences between the groups were analyzed by analysis of variance, followed by a least significant difference test. $P<0.05$ was considered to indicate a statistically significant difference.

\section{Results}

S. boulardii ameliorates the symptoms of DSS-induced colitis in mice. The effects of S. boulardii in mice with DSS-induced 
A

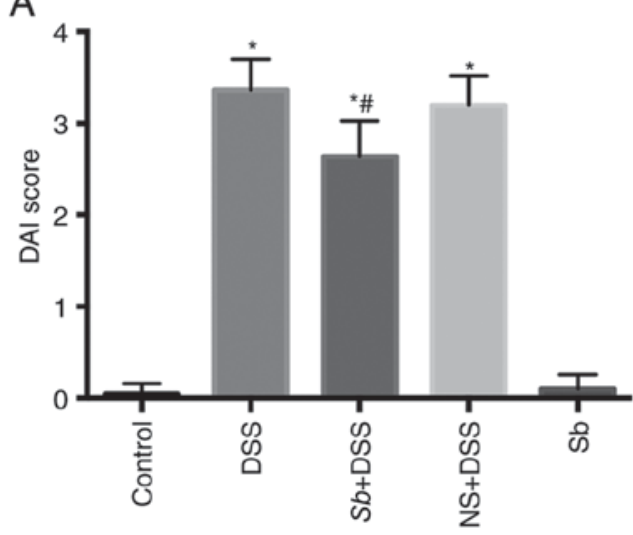

$\mathrm{B}$

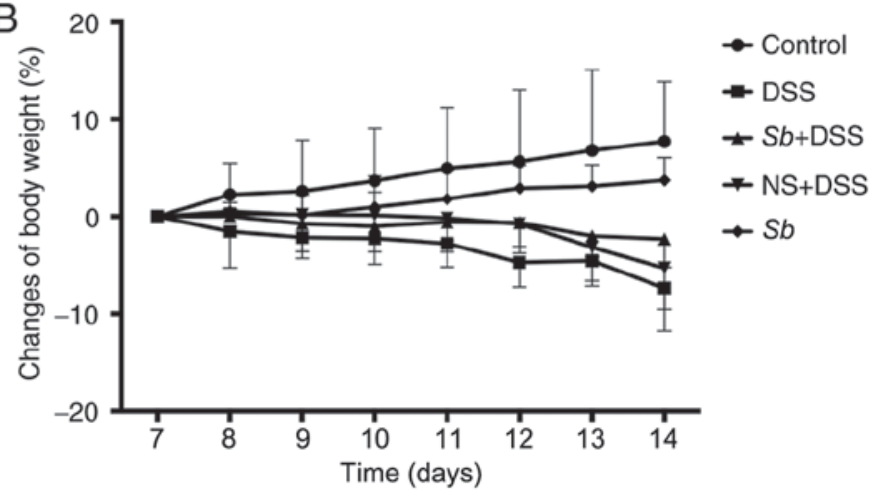

Figure 1. Effects of Saccharomyces boulardii on the clinical symptoms in mice with DSS-induced colitis. (A and B) Mice were orally administered S. boulardii $(150 \mathrm{mg} / \mathrm{kg}$ ) for 14 consecutive days and treated with DSS from day 8 , and the effects of $S$. boulardii on clinical symptoms in DSS-induced colitis was assessed by measuring the alterations of body weight and DAI. (A) DAI scores at day 14. Sb co-treatment reduced DAI scores in mice with DSS-induced colitis. (B) Alterations in body weight at days 7-14. Sb ameliorated weight loss in mice with DSS-induced colitis. Results are presented as the mean \pm standard deviation; $\mathrm{n}=10 ;{ }^{*} \mathrm{P}<0.05$ vs. Control; ${ }^{~} \mathrm{P}<0.05$ vs. DSS. DAI, disease activity index; DSS, dextran sulfate sodium; NS, normal saline; Sb, Saccharomyces boulardii.

colitis were investigated. The mice treated with DSS developed clinical signs of colitis, which included anorexia, lethargy, diarrhea, rectal bleeding and a loss of body weight. Whether pretreatment with $S$. boulardii improved the clinical symptoms of DSS-induced colitis was investigated. Mice in the $S b+\mathrm{DSS}$ group were administered $S b(150 \mathrm{mg} / \mathrm{kg}, 0.2 \mathrm{ml})$ by gavage for 14 consecutive days and DSS was administered in the water from day 8 . Mice in the NS+DSS group were administered $0.2 \mathrm{ml}$ NS by gavage as the negative control. DSS treatment significantly increased the DAI score and resulted in notable weight loss compared with the Control-treated mice $(\mathrm{P}<0.05$; Fig. 1A and B, respectively). NS-treatment alone did not significantly alter the symptoms of DSS-induced colitis or weight loss (Fig. 1). S. boulardii treatment significantly decreased DAI scores and reduced the weight loss induced by DSS in the $S b+\mathrm{DSS}$ mice compared with mice in the DSS group $(\mathrm{P}<0.05$; Fig. 1). Additionally, administration of $S$. boulardii alone exhibited no effect on body weight and DAI score $(\mathrm{P}>0.05)$, which indicated that $S$. boulardii was safe to administer to mice. These results demonstrated that oral administration of $S$. boulardii may ameliorate the symptoms of DSS-induced colitis in mice.

S. boulardii reduces histological damage in colitis model mice. $\mathrm{H} \& \mathrm{E}$ staining of colon tissues was performed to analyze the histological features of DSS-induced colitis (Fig. 2A). The Control and $S b$ groups exhibited healthy intestinal mucosa with no inflammatory infiltration in the mucosal, submucosal or muscular layers (Fig. 2A), which suggested that daily $S$. boulardii treatment did not alter gut mucosal morphology. Histological examination of colons from mice with DSS-induced colitis exhibited multifocal areas of mucosal erosion, epithelial cell injury and significant mucosal infiltration of neutrophils (Fig. 2A). However, these histological signs were reduced in mice that were treated with $S$. boulardii, with a reduction in the inflammatory activity and neutrophil infiltration. These results suggested that $S$. boulardii may suppress the development of inflammation induced by DSS treatment. Furthermore, histological scoring was performed for histological scoring of IBD severity (Fig. 2B). The histological scores of the DSS-treated group were significantly increased compared with the Control at day 14, whereas the histological score was significantly reduced in the $S b+\mathrm{DSS}$ group compared with the DSS-only group ( $\mathrm{P}<0.05$; Fig. $2 \mathrm{~B})$; no significant difference was identified in the NS+DSS group compared with DSS-only.

S. boulardii protects the colon mucosal barrier in mice with DSS-induced colitis. Destruction of the colon mucosal barrier serves an important role in the development of IBD (17). To determine whether $S$. boulardii protected the colon mucosal barrier, the expression of tight junction proteins were examined, including occludin and claudin-1, which are important for colon mucosal barrier function. Results from RT-qPCR demonstrated that the expression of occludin mRNA was decreased in the DSS-treated groups compared with the Control group ( $\mathrm{P}<0.05$; Fig. 3A). Colitis model mice co-treated with $S$. boulardii exhibited a significant increase in occludin mRNA expression levels compared with DSS-only treated mice $(\mathrm{P}<0.05)$; treatment with $S$. boulardii alone had no effect on occludin mRNA expression. There was no significant difference in claudin-1 mRNA expression levels in the colon tissues of mice with DSS-induced colitis ( $\mathrm{P}<0.05$; Fig. 3B); however, whereas co-treatment with $S$. boulardii significantly increased claudin-1 expression levels compared with the Control and DSS groups. Similar effects were observed in the $S b$-only treated mice. The protein distribution of occludin and claudin-1 was investigated by immunohistochemistry. $S$. boulardii ameliorated the DSS-induced decrease in occludin and claudin-1 in the adjacent epithelial cells of the colon epithelium of mice with DSS-induced colitis (Fig. 3C). The protein expression levels of occludin and claudin-1 were analyzed by western blotting (Fig. 3D). S. boulardii co-treatment ameliorated the decrease in occludin and claudin-1 protein levels in the colon of mice with DSS-induced colitis, with a significant difference in occludin expression (Fig. 3D). In the $S b+\mathrm{DSS}$ group, claudin-1 mRNA expression was significantly higher compared with the Control and DSS 
A

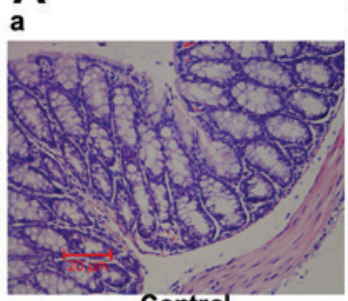

Control

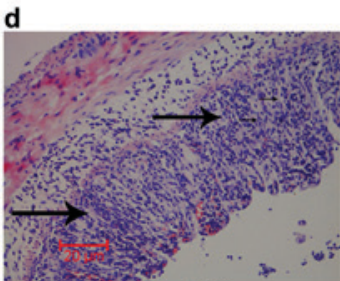

NS+DSS b

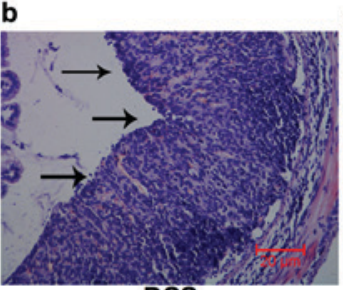

DSS

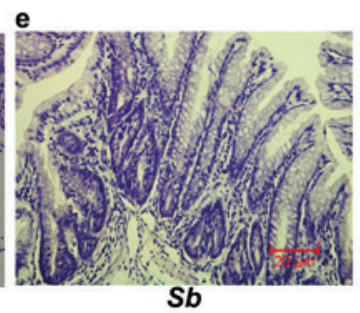

B

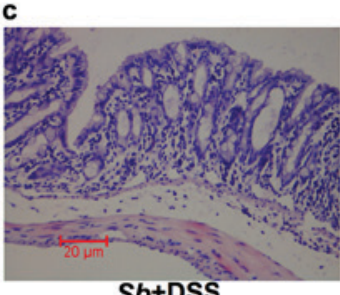

Sb+DSS

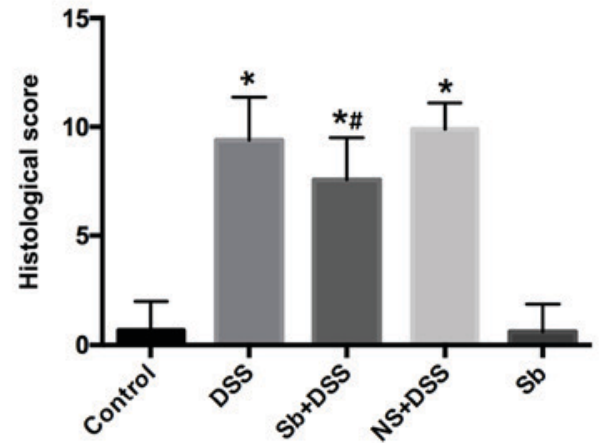

Figure 2. Effects of Saccharomyces boulardii on histological damage in colitis model mice. (A) Histological features of the colon tissues at day 14 in (a) the Control group; (b) the DSS group; (c) the $S b+\mathrm{DSS}$ group; (d) the NS+DSS group; and (e) the $S b$ group; magnification, x200. (B) The histological scores were calculated and presented as the mean \pm standard deviation; $\mathrm{n}=10$. S. boulardii ameliorated histological damage and reduced histological scores in mice with DSS-induced colitis. " $\mathrm{P}<0.05$ vs. Control; ${ }^{\#} \mathrm{P}<0.05$ vs. DSS. DSS, dextran sulfate sodium; NS, normal saline; Sb, Saccharomyces boulardii.

A

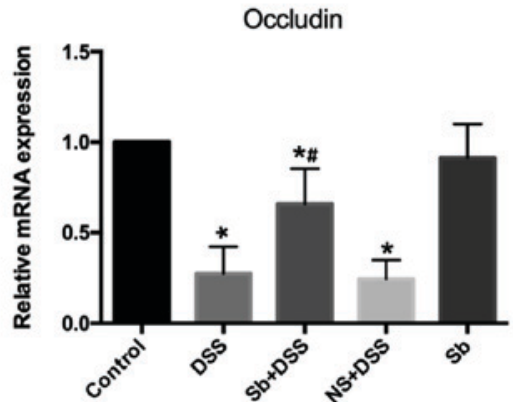

B

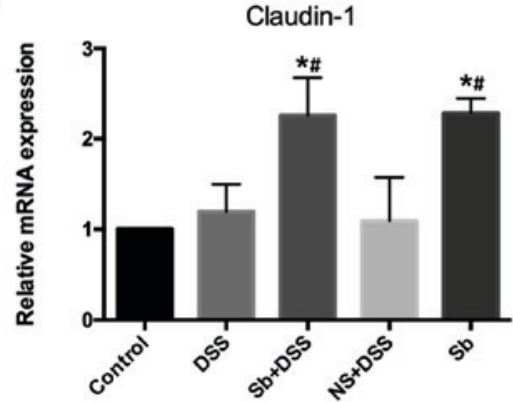

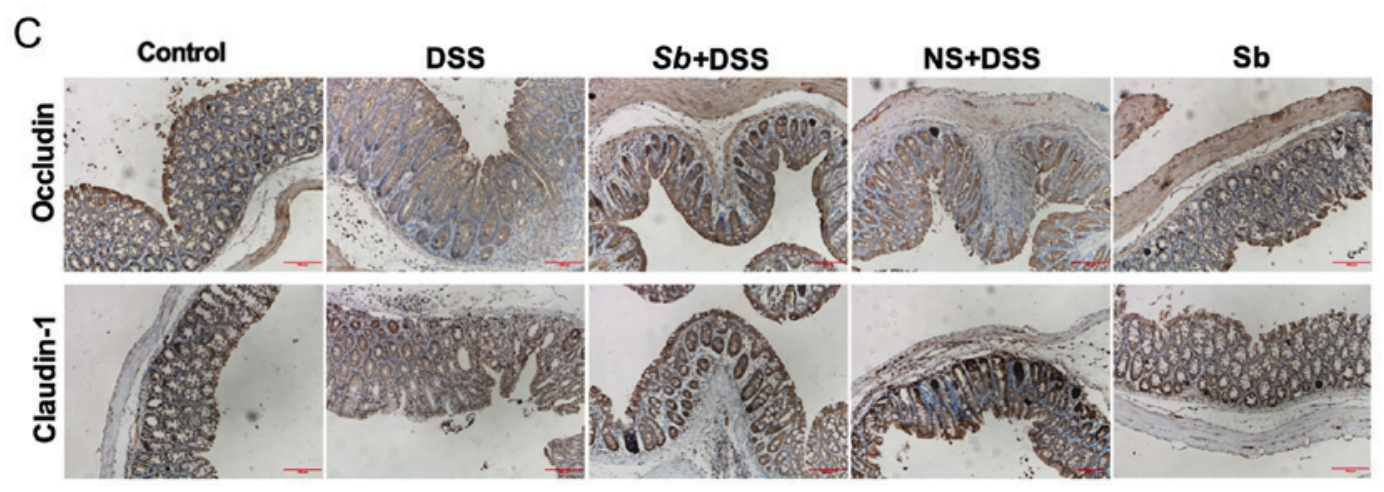
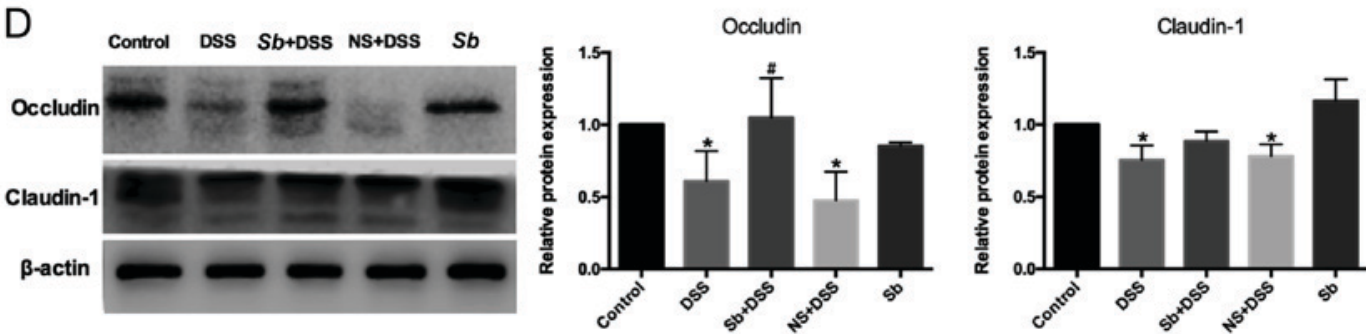

Figure 3. Effects of Saccharomyces boulardii on the colon mucosal barrier in colitis model mice. mRNA expression levels of (A) occludin and (B) claudin-1 in colonic tissues was measured by reverse transcription-quantitative polymerase chain reaction. Results were reported as fold-change relative to the Control group and expressed as the mean \pm standard deviation; $\mathrm{n}=10 ;{ }^{*} \mathrm{P}<0.05$ vs. Control group; ${ }^{*} \mathrm{P}<0.05$ vs. DSS. (C) The protein distribution of occludin and claudin- 1 was examined by immunohistochemistry. Scale bar $=50 \mu \mathrm{m}$. (D) Protein expression levels of occludin and claudin-1 were analyzed by western blotting; $\beta$-actin was used as a loading control and for normalization. Results are presented as the mean \pm standard deviation; $\mathrm{n}=10$; ${ }^{*} \mathrm{P}<0.05$ vs. Control; ${ }^{*} \mathrm{P}<0.05$ vs. DSS. DSS, dextran sulfate sodium; NS, normal saline; Sb, Saccharomyces boulardii. 
A

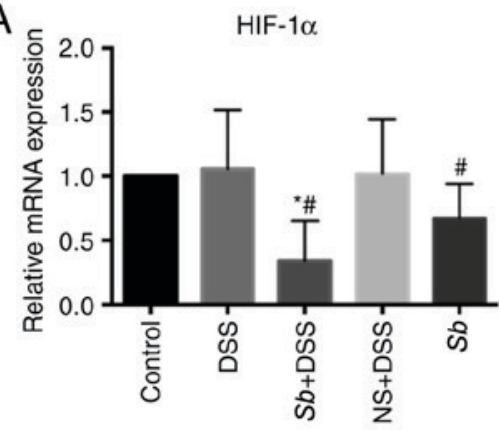

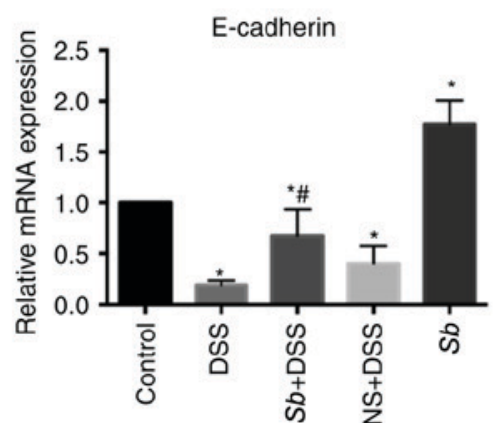

Sb+DSS

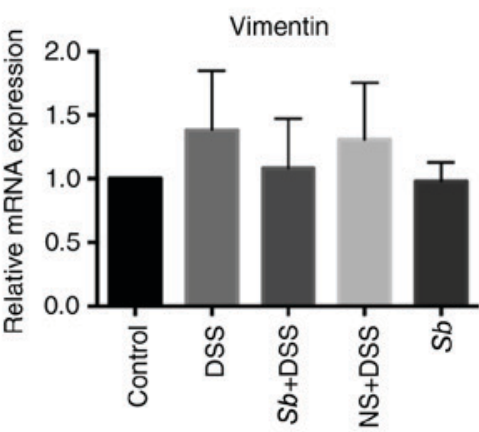

NS+DSS

$\mathrm{Sb}$

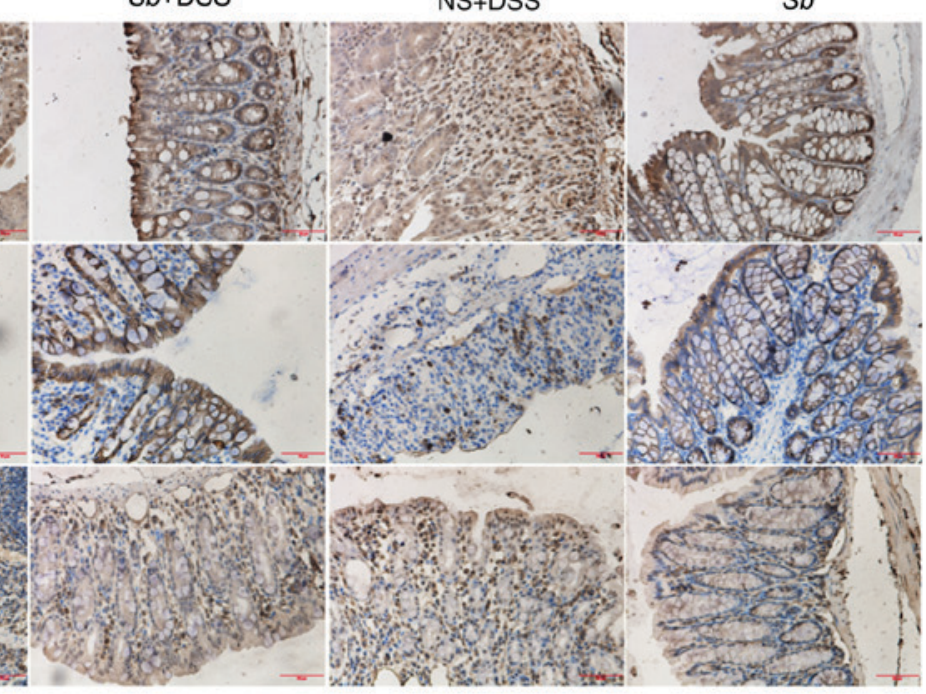

B

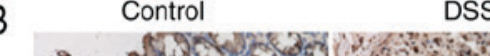

DS
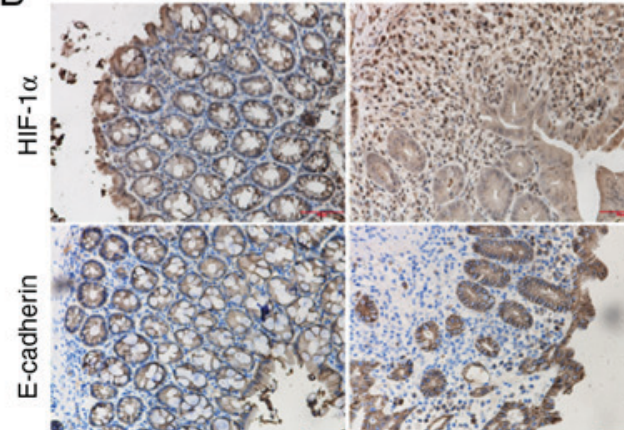

\&.

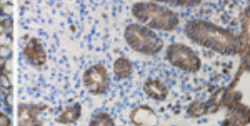

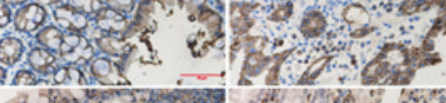

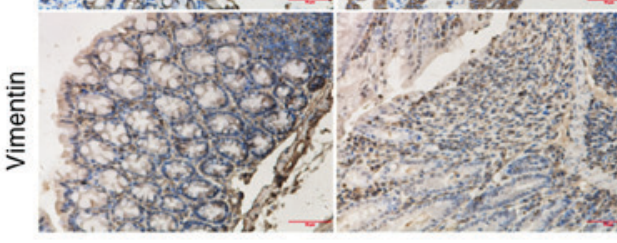

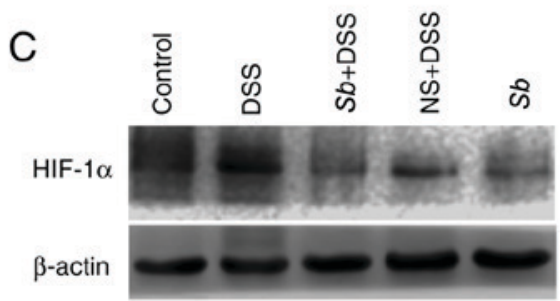

$\mathrm{HIF}-1 \alpha$

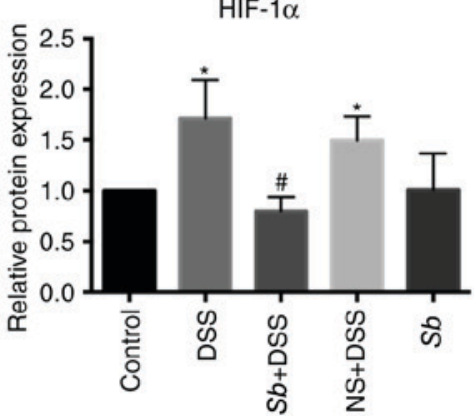

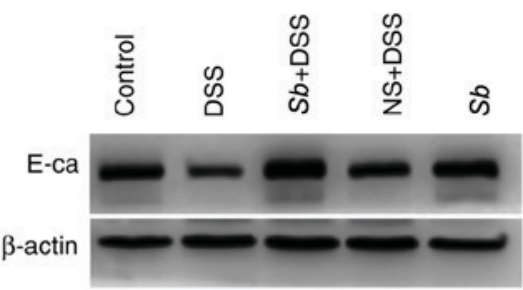

E-cadherin

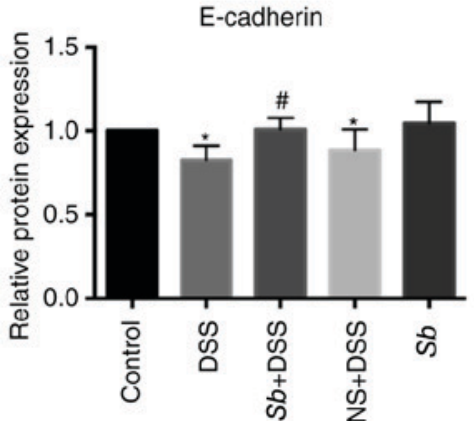

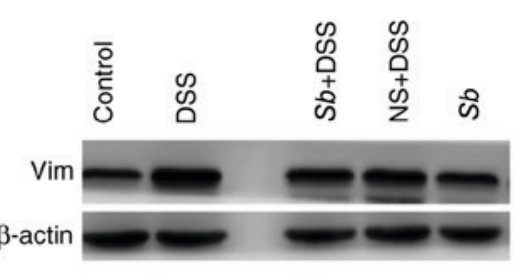

Vimentin

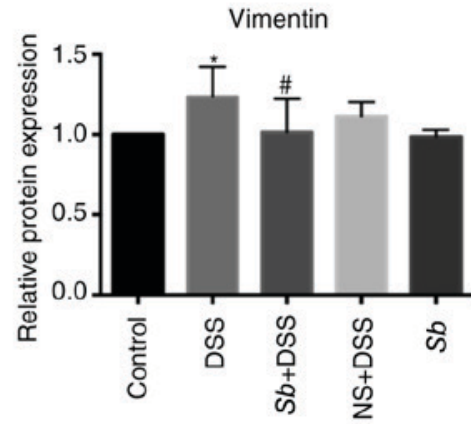

Figure 4. Effects of Saccharomyces boulardii on HIF-1 $\alpha$ and epithelial-mesenchymal transition-associated marker expression in the colon tissue of colitis model mice. (A) mRNA expression levels of HIF-1 $\alpha$, E-ca and Vim in the colon tissues were measured by reverse transcription quantitative polymerase chain reaction. Results were reported as fold-change relative to the control group and expressed as the mean \pm standard deviation; $\mathrm{n}=10$; ${ }^{*} \mathrm{P}<0.05 \mathrm{vs}$. Control; ${ }^{\sharp} \mathrm{P}<0.05$ vs. DSS. (B) Protein distribution of HIF-1 $\alpha$, E-ca and Vim as detected by immunohistochemistry. (C) Protein expression levels of HIF-1 $\alpha$, E-ca and Vim were analyzed by western blotting; $\beta$-actin was used as a loading control and for normalization. Data are presented as the mean \pm standard deviation; $\mathrm{n}=10$; ${ }^{*} \mathrm{P}<0.05$ vs. Control; "P<0.05 vs. DSS. DSS, dextran sulfate sodium; E-ca, epithelial cadherin; HIF, hypoxia inducible factor; NS, normal saline; Sb, Saccharomyces boulardii; Vim, vimentin.

group, whereas no significant differences were observed in claudin-1 protein expression levels between the same groups. This suggested that DSS damages claudin-1 at the protein level.
Effects of S. boulardii on the expression of HIF-1 $\alpha$ and several EMT-associated markers in the colon tissues of colitis model mice. HIF-1 $\alpha$ serves an important and complex regulatory role in the course of IBD (18). To investigate whether S. boulardii 

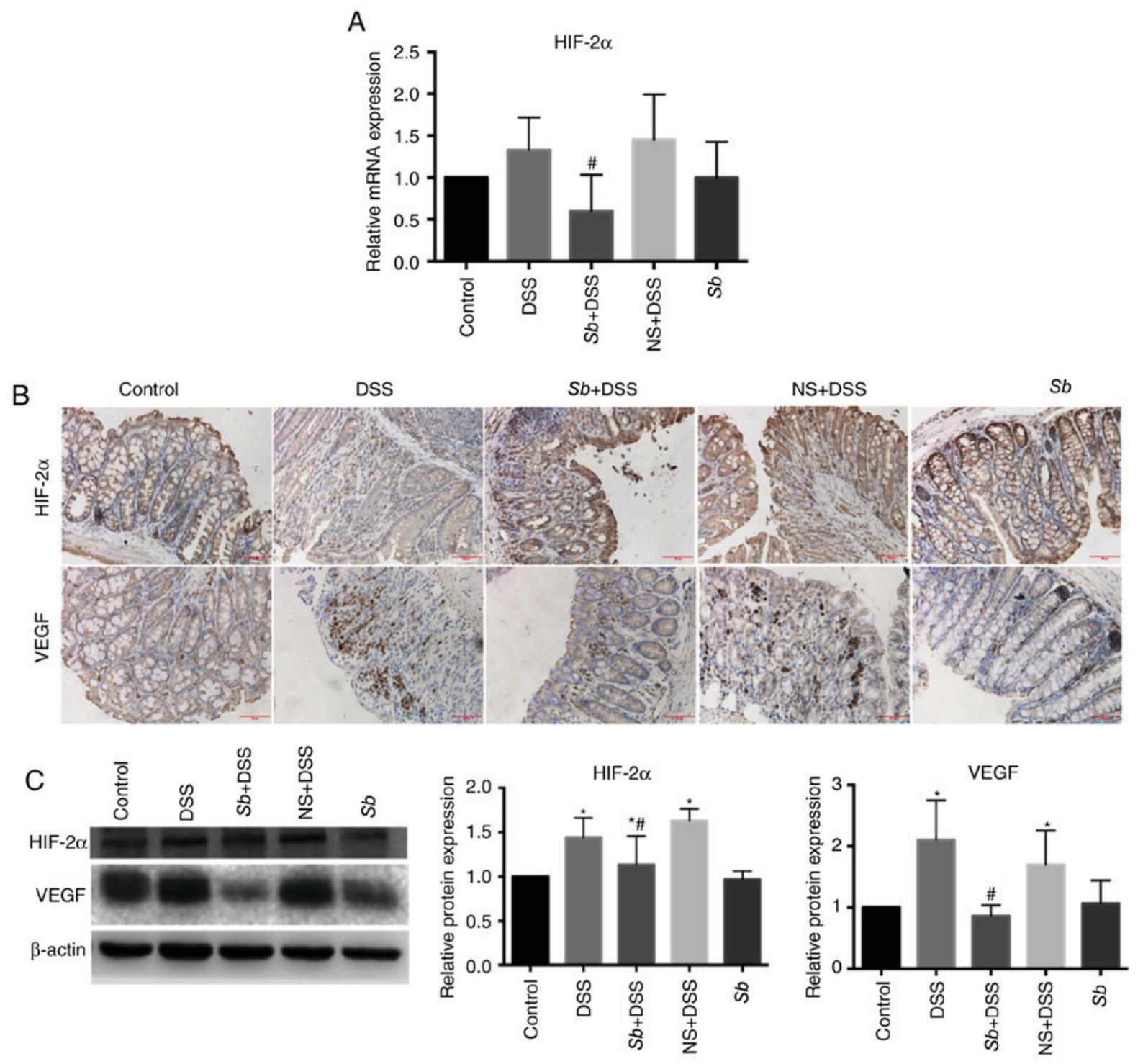

Figure 5. Effects of Saccharomyces boulardii on HIF-2 $\alpha$ and VEGF expression in the colon tissue samples of colitis model mice. (A) mRNA expression levels of HIF- $2 \alpha$ in the colon tissues were measured by reverse transcription-quantitative polymerase chain reaction. Results were reported as fold-change relative to the Control group and expressed as the mean \pm standard deviation; $\mathrm{n}=10 ;{ }^{*} \mathrm{P}<0.05$ vs. DSS. (B) Protein distribution of HIF- $2 \alpha$ and VEGF as detected by immunohistochemistry. Scale bar $=50 \mu \mathrm{m}$. (C) Protein expression levels of HIF-2 $\alpha$ and VEGF were analyzed by western blotting; $\beta$-actin was used as a loading control and for normalization. Data are expressed as the mean \pm standard deviation; $n=10 ;{ }^{*} \mathrm{P}<0.05$ vs. Control; $\mathrm{P}<0.05$ vs. DSS. DSS, dextran sulfate sodium; HIF, hypoxia inducible factor; NS, normal saline; Sb, Saccharomyces boulardii; VEGF, vascular endothelial growth factor.

affected HIF-1 $\alpha$, HIF-1 $\alpha$ expression levels were examined in the colon tissues of mice. Results from RT-qPCR demonstrated no significant differences in the mRNA expression levels of HIF-1 $\alpha$ in the colon tissues of the DSS group compared with the Control group (Fig. 4A); similar results were observed in the NS+DSS mice. Conversely, HIF-1 $\alpha$ mRNA expression levels in the $S b+$ DSS and $S b$ groups were significantly decreased compared with the Control and DSS-only groups $(\mathrm{P}<0.05$; Fig. 4A). Immunohistochemical analysis demonstrated a dispersed distribution of HIF-1 $\alpha$ in the colon tissues of the Control and $S b$ groups (Fig. 4B). However, the nuclear HIF-1 $\alpha$ staining was significantly increased in the DSS and NS+DSS groups, compared with the Control tissues $(\mathrm{P}<0.05)$; whereas HIF-1 $\alpha$ expression in the colon tissues of the $S b+\mathrm{DSS}$ group was decreased compared with the DSS group. Western blotting demonstrated that HIF-1 $\alpha$ protein expression levels in the colon tissues of mice in the DSS and NS+DSS groups was significantly increased compared with the Control group $(\mathrm{P}<0.05)$, whereas expression in the $S b+\mathrm{DSS}$ group was significantly decreased compared with the DSS group $(\mathrm{P}<0.05$; Fig. 4C).

HIF-1 $\alpha$ promotes the EMT process of colonic epithelial cells (19); therefore, the mRNA and protein distribution and quantification of EMT markers (E-cadherin and vimentin) were further examined in the colon tissues. Results from RT-qPCR demonstrated that the expression levels of E-cadherin mRNA in the DSS and NS+DSS groups were significantly decreased compared with the Control group $(\mathrm{P}<0.05$; Fig. 4A), whereas $S$. boulardii co-treatments significantly decreased E-cadherin mRNA expression; the expression of E-cadherin mRNA was significantly increased in the $S b$ group compared with the Control group $(\mathrm{P}<0.05)$. The expression of vimentin mRNA in the colon tissues of the DSS and NS+DSS groups appeared to be increased compared with the Control group, 
but the difference was not significant (Fig. 4A). S. boulardii co-treatment notably reduced the DSS-induced increase in vimentin mRNA expression.

Immunohistochemistry demonstrated that the E-cadherin protein expression in the colon tissues of the Control and $\mathrm{Sb}$ groups was mainly distributed between the surface and the crypt of the intestinal epithelium, whereas expression in the DSS and NS+DSS groups exhibited different degrees of expression deletion, discontinuous distribution and was completely absent in certain areas (Fig. 4B). E-cadherin protein expresion in the colon tissues of the $S b+\mathrm{DSS}$ group exhibited some degree of deletion; however, this was to a lower degree compared with the DSS group. Vimentin is mainly expressed in the cytoplasm of interstitial cells. The expression of vimentin in the DSS and NS+DSS groups was notably increased compared with the Control group (Fig. 4B). The expression of vimentin in the $\mathrm{S} b+\mathrm{DSS}$ group was increased compared with the Control, but notably decreased compared with the DSS group (Fig. 4B).

Western blotting demonstrated that the expression levels of E-cadherin in the colon tissues of the DSS and NS+DSS groups were significanlty decreased compared with the Control group $(\mathrm{P}<0.05$; Fig. 4C). However, expression of E-cadherin in the colon tissue of the $S b+\mathrm{DSS}$ group was significantly increased compared with the DSS group ( $\mathrm{P}<0.05$; Fig. 4C). Expression of vimentin in the colon tissue of the DSS and NS+DSS groups was significantly increased compared with the Control group $(\mathrm{P}<0.05$; Fig. 4C), whereas expression of vimentin in the $\mathrm{S} b+\mathrm{DSS}$ group was significantly decreased compared with the DSS group ( $\mathrm{P}<0.05$; Fig. 4C).

Taken together, these data indicated that administration of S. boulardii reduced expression of HIF-1 $\alpha$ and affected the expression of several EMT-associated markers during experimental colitis.

Effect of S. boulardii on HIF-2 $\alpha$ and VEGF expression in colon tissues of colitis model mice. HIF-2 $\alpha$ is an important transcription factor in the pathogenesis of colitis and may increase the expression of proinflammatory factors (2). To investigate whether $S$. boulardii affected HIF- $2 \alpha$, HIF- $2 \alpha$ expression levels in the colon tissues of mice were examined. Results from RT-qPCR demonstrated that the mRNA expression levels of HIF- $2 \alpha$ were notably increased in the DSS and NS+DSS groups compared with the Control group, but the differences were not significant ( $\mathrm{P}>0.05$; Fig. 5A). Expression of HIF- $2 \alpha$ mRNA in colon tissues of the $S b+\mathrm{DSS}$ group was significantly decreased compared with the DSS group. There was no significant difference identified in HIF- $2 \alpha$ mRNA expression between the $S b$ and Control groups. Immunohistochemical analysis demonstrated a diffuse distribution of HIF-2 $\alpha$ expression in the colon tissues of the Control and $S b$ groups (Fig. 5B). However, nuclear HIF-2 $\alpha$ staining was notably increased in the DSS and NS+DSS groups; HIF- $2 \alpha$ staining in the colon tissues of the $S b+\mathrm{DSS}$ group was markedly decreased compared with the DSS group. Western blotting demonstrated that the protein expression levels of HIF- $2 \alpha$ in the colon tissue of the DSS and NS+DSS groups were significantly increased compared with expression in the Control group. HIF-2 $\alpha$ protein expression levels in the colon tissues of the $S b+\mathrm{DSS}$ group were significantly decreased compared with the DSS group $(\mathrm{P}<0.05$; Fig. 5C).
HIF-2 $\alpha$ regulates expression of VEGF, which is the main regulator of hypoxia-induced neovascularization (20). Protein distribution and quantification of VEGF was examined. Immunohistochemical analysis demontrated that VEGF was expressed in the cytoplasm of neovascular endothelial cells (Fig. 5B). Expression of VEGF in the colon tissue of the Control group was low, but was markedly increased in the DSS and NS+DSS groups (Fig. 5B). Expression of VEGF in the $S b+\mathrm{DSS}$ group was notably decreased compared with the DSS group. VEGF expression in the $S b$ group was similar to that in the Control group. Western blotting demonstrated that the expression of VEGF in the colon tissues of the Control group was low, whereas protien expression levels of VEGF in the DSS and NS+DSS groups was significantly increased comapared with the Control ( $\mathrm{P}<0.05$; Fig. 5C). VEGF expression levels in the $S b+\mathrm{DSS}$ group was significantly decreased compared with the DSS group $(\mathrm{P}<0.05)$; the expression of VEGF in the $S b$ group was similar to that in the Control group.

\section{Discussion}

IBD is a serious health problem worldwide. Although pharmaceutical approaches to disease control have improved in recent years, there remain a series of problems including adverse effects, poor patient compliance and relapse. Therefore, effective and improved strategies are urgently needed to treat IBD.

Probiotics are defined as living microorganisms that, when administered in adequate amounts, confer a health benefit to the host (21). Probiotics have been investigated as a therapeutic approach in a range of disorders, including IBD and other intestinal problems $(10,22)$. S. boulardii, a non-pathogenic probiotic yeast, has been used worldwide for several decades to protect against intestinal injury and inflammation (9). One previous study demonstrated that daily use of 750-1,000 mg $S$. boulardii, with continuous application for 6 months, significantly reduced the recurrence of Crohn's disease (13). However, the underlying mechanism is complex and not completely understood. In the present study, $S$. boulardii was used to determine its bioactivity in mice with DSS-induced colitis. Experimental colitis induced by DSS in mice is thought to share a number of important characteristics with forms of human IBD. It has been demonstrated that administration of DSS in mice leads to body weight loss, epithelial cell inflammation, mucosal ulceration, neutrophil infiltration, colon shortening and bloody diarrhea, which are similar to the signs observed in ulcerative colitis in humans (15).

The present study examined the general condition of the colitis model mice, as well as alterations in body weight, and DAI and histological scores. DAI and histological scores were the highest in the DSS group, and infiltration of inflammatory cells was similar to that of ulcerative colitis. DAI and histological scores in the NS+DSS groups were similar to those in the DSS group, indicating that NS alone did not alter the symptoms of DSS-induced colitis. The $S b$-only group demonstrated no effects on body weight, no microscopic alterations in the colon tissue and no adverse effects, which indicated that $S$. boulardii supplementation did not negatively affect mice. Model mice co-treated with $S b$ demonstrated that $S$. boulardii may alleviate colitis symptoms, including intestinal bleeding, loose stools and body weight loss, and may reduce damage of the colon tissue. 
Destruction of the colon mucosal barrier serves an important role in the development of IBD (17). The expression of tight junction proteins, including occludin and claudin-1, was examined by RT-qPCR, immunohistochemistry and western blotting. S. boulardii demonstrated a protective effect on the colon mucosal barrier. This is consistent with the results of a previous study (23).

The underlying mechanism was further examined in the present study, as the precise etiology of IBD is unknown. Hypoxia serves an important role in the inflammatory and injury response (2). Hypoxic stress that occurs when cellular oxygen demand is higher than its supply is common in tissues faced with infection and inflammation (24). The HIF complex is a key transcription factor for cellular adaption to low oxygen tension (25). HIF-1 $\alpha$ and HIF-2 $\alpha$ can bind to the same canonical hypoxia response elements; however, they regulate a distinct subset of genes. HIFs serve an important role in adaptation to the hypoxic environment, but may also lead to metabolic disorders and a variety of pathophysiological alterations (2). One previous study demonstrated that HIF-1 $\alpha$ and HIF- $2 \alpha$ are strongly activated in IBD patients (2). The present study examined whether $S$. boulardii affected HIFs. The results indicated that HIF-1 $\alpha$ was activated by inflammation. This is consistent with previous studies $(2,4,26)$. The results also indicated that $S$. boulardii reduced inflammation by inhibiting expression of HIF-1 $\alpha$. Expression of HIF-1 $\alpha$ mRNA did not increase in the DSS group, indicating that DSS may regulate HIF-1 $\alpha$ at the protein rather than transcription level. However, HIF- $1 \alpha$ mRNA expression in the $S b+$ DSS group was decreased compared with the Control group, indicating that $S$. boulardii reduced the protein level of HIF-1 $\alpha$ by affecting its mRNA expression levels.

Hypoxia can promote cell EMT through a variety of signaling pathways (27). EMT is a dynamic biological process in which polarized epithelial cells lose their epithelial characteristics and exhibit phenotypes of mesenchymal cells. A previous study demonstrated that EMT serves an important role in the pathogenesis of IBD (28). Therefore, inhibition of EMT has the potential to improve the clinical symptoms of IBD. Hypoxia-stabilized HIF-1 $\alpha$ has been demonstrated to upregulate EMT-associated transcription factors, including TWIST and Snail $(29,30)$ indicating that HIF-1 $\alpha$ serves a critical role in hypoxia-induced EMT. E-cadherin and vimentin expression was examined in the present study to evaluate EMT progression. mRNA and protein expression levels of E-cadherin and vimentin were altered. Expression of E-cadherin mRNA and protein in the DSS group was decreased compared with the Control group. This is consistent with previous studies $(31,32)$. However, co-treatment with $S$. boulardii ameliorated this reduction. Expression of vimentin mRNA and protein in the DSS group was increased compared with the Control group. This is consistent with previous studies $(33,34)$. However, $S$. boulardii co-treatment ameliorated this increase. The present study indicated that $S$. boulardii ameliorated EMT of the colon tissue epithelial cells. Since EMT serves an important role in the pathogenesis of IBD, inhibition of EMT has the potential to improve the clinical symptoms of IBD, thereby controlling inflammation.

HIF- $2 \alpha$ is a transcription factor that activates inflammatory mediators in the colon epithelium, including tumor necrosis factor- $\alpha$, to promote the development of colitis in mice (35). In the present study, expression of HIF-2 $\alpha$ protein in colon tissue of the DSS group was increased compared with the Control group, which indicated that HIF-2 $\alpha$ was activated by inflammation. Expression of HIF- $2 \alpha$ mRNA and protein in the colon tissues of the $S b+\mathrm{DSS}$ group was decreased compared with the DSS group, indicating that $S$. boulardii may reduce inflammation by inhibiting expression of HIF- $2 \alpha$.

During inflammation and hypoxia, angiogenesis is induced to compensate for poor oxygenation; however, this may result in aberrant vasculature and contribute to the pathogenesis of chronic inflammation (24). VEGF can promote neovascularization. A previous study demonstrated that VEGF is an important mediator for IBD and promotes small bowel angiogenesis and inflammation (36). Overexpression of VEGF in DSS-induced colitis may aggravate the condition, whereas overexpression of soluble VEGF receptor, which can block VEGF, is beneficial. Therefore, inhibition of the VEGF/VEGF receptor pathway can reduce intestinal inflammation in IBD patients (37). HIF-2 $\alpha$ regulates expression of VEGF and is a major regulator of hypoxia-induced neovascularization $(38,39)$. In the present study, expression of VEGF protein in the DSS group was increased compared with the Control group, and $S$. boulardii co-treatment ameliorated this increase. $S$. boulardii may reduce VEGF expression in the DSS-induced colitis to a certain extent, thereby reducing inflammation.

In conclusion, the present study demonstrated that $S$. boulardii may alleviate DSS-induced colitis, inhibit the expression of HIF- $1 \alpha$ and HIF- $2 \alpha$, and inhibit EMT and VEGF expression. It was hypothesized that $S$. boulardii may inhibit EMT progression via reducing HIF-1 $\alpha$ expression in DSS-treated mice. However, more detailed studies are needed to further elucidate the specific mechanism of HIF- $1 \alpha$ regulating EMT progression in IBD, including in vivo and in vitro experiments. By observing the effect of $S$. boulardii on HIFs, our study may reveal a new mechanism by which $S$. boulardii yeast works. Further investigations are necessary to identify the underlying mechanism of the therapeutic effects of S. boulardii, in order to improve IBD treatment.

\section{Acknowledgements}

The authors would like to thank the Department of Pharmacology, School of Pharmaceutical Sciences, China Medical University (Shenyang, China), for help with experimental methods and the provision of experimental equipment.

\section{Funding}

The present study was supported by The Natural Science Foundation of Liaoning Province (grant no. 2015020515).

\section{Availability of data and materials}

The data used and/or analyzed during the current study are available from the corresponding author on reasonable request. 


\section{Authors' contributions}

MJS conceived and designed the project; HZ performed the experiments; HJZ, LG, YZ and YL analyzed the data. HZ wrote the manuscript. All authors have read and approved the final manuscript.

\section{Ethics approval and consent to participate}

All animal experiments were performed in accordance with the Animals Scientific Procedures Act (1986) and were approved by the Ethics Review Committee for Animal Experimentation of the China Medical University. All animal studies comply with the ARRIVE guidelines (http://www.nc3rs.org.uk/arrive-guidelines) and the AVMA euthanasia guidelines 2013 (AVMA euthanasia guidelines 2013.pdf).

\section{Patient consent for publication}

Not applicable.

\section{Competing interests}

The authors declare they have no competing interests.

\section{References}

1. Molodecky NA, Soon IS, Rabi DM, Ghali WA, Ferris M, Chernoff G, Benchimol EI, Panaccione R, Ghosh S, Barkema HW, et al: Increasing incidence and prevalence of the inflammatory bowel diseases with time, based on systematic review. Gastroenterology 142: 46-54 e42; quiz e30, 2012

2. Shah YM: The role of hypoxia in intestinal inflammation. Mol Cell Pediatr 3: 1, 2016.

3. Fagundes RR and Taylor CT: Determinants of hypoxia-inducible factor activity in the intestinal mucosa. J Appl Physiol 123: 1328-1334, 2017

4. Cummins EP and Crean D: Hypoxia and inflammatory bowel disease. Microbes Infect 19: 210-221, 2017.

5. Reiff $C$ and Kelly D: Inflammatory bowel disease, gut bacteria and probiotic therapy. Int J Med Microbiol 300: 25-33, 2010.

6. Guandalini S: Update on the role of probiotics in the therapy of pediatric inflammatory bowel disease. Expert Rev Clin Immunol 6: 47-54, 2010.

7. Yu CG and Huang Q: Recent progress on the role of gut microbiota in the pathogenesis of inflammatory bowel disease. J Dig Dis 14: 513-517, 2013.

8. Hold GL, Smith M, Grange C, Watt ER, El-Omar EM and Mukhopadhya I: Role of the gut microbiota in inflammatory bowel disease pathogenesis: What have we learnt in the past 10 years? World J Gastroenterol 20: 1192-1210, 2014.

9. Hatoum R, Labrie S and Fliss I: Antimicrobial and probiotic properties of yeasts: From fundamental to novel applications. Front Microbiol 3: 421, 2012.

10. Generoso SV, Viana M, Santos R, Martins FS, Machado JA, Arantes RM, Nicoli JR, Correia MI and Cardoso VN: Saccharomyces cerevisiae strain UFMG 905 protects against bacterial translocation, preserves gut barrier integrity and stimulates the immune system in a murine intestinal obstruction model. Arch Microbiol 192: 477-484, 2010.

11. Buts JP and De Keyser N: Effects of Saccharomyces boulardii on intestinal mucosa. Dig Dis Sci 51: 1485-1492, 2006.

12. Kelesidis T and Pothoulakis C: Efficacy and safety of the probiotic Saccharomyces boulardii for the prevention and therapy of gastrointestinal disorders. Therap Adv Gastroenterol 5: 111-125, 2012.

13. Guslandi M, Mezzi G, Sorghi M and Testoni PA: Saccharomyces boulardii in maintenance treatment of Crohn's disease. Dig Dis Sci 45: 1462-1464, 2000.
14. Hamamoto N, Maemura K, Hirata I, Murano M, Sasaki S and Katsu K: Inhibition of dextran sulphate sodium (DSS)-induced colitis in mice by intracolonically administered antibodies against adhesion molecules (endothelial leucocyte adhesion molecule-1 (ELAM-1) or intercellular adhesion molecule-1 (ICAM-1)). Clin Exp Immunol 117: 462-468, 1999.

15. Cooper HS, Murthy SN, Shah RS and Sedergran DJ: Clinicopathologic study of dextran sulfate sodium experimental murine colitis. Lab Invest 69: 238-249, 1993.

16. Livak KJ and Schmittgen TD: Analysis of relative gene expression data using real-time quantitative PCR and the 2(-Delta Delta C(T)) Method. Methods 25: 402-408, 2001.

17. McCole DF: IBD candidate genes and intestinal barrier regulation. Inflamm Bowel Dis 20: 1829-1849, 2014.

18. Kim YE, Lee M, Gu H, Kim J, Jeong S, Yeo S, Lee YJ, Im SH, Sung YC, Kim HJ, et al: HIF-1 $\alpha$ activation in myeloid cells accelerates dextran sodium sulfate-induced colitis progression in mice. Dis Model Mech 11: 11, 2018.

19. Kim SL, Park YR, Lee ST and Kim SW: Parthenolide suppresses hypoxia-inducible factor-1 $\alpha$ signaling and hypoxia induced epithelial-mesenchymal transition in colorectal cancer. Int J Oncol 51: 1809-1820, 2017.

20. Feng N, Chen H, Fu S, Bian Z, Lin X, Yang L, Gao Y, Fang J and Ge Z: HIF- $1 \alpha$ and HIF- $2 \alpha$ induced angiogenesis in gastrointestinal vascular malformation and reversed by thalidomide. Sci Rep 6: 27280, 2016.

21. Hill C, Guarner F, Reid G, Gibson GR, Merenstein DJ, Pot B, Morelli L, Canani RB, Flint HJ, Salminen S, et al: Expert consensus document. The International Scientific Association for Probiotics and Prebiotics consensus statement on the scope and appropriate use of the term probiotic. Nat Rev Gastroenterol Hepatol 11: 506-514, 2014.

22. Generoso SV, Viana ML, Santos RG, Arantes RM, Martins FS, Nicoli JR, Machado JA, Correia MI and Cardoso VN: Protection against increased intestinal permeability and bacterial translocation induced by intestinal obstruction in mice treated with viable and heat-killed Saccharomyces boulardii. Eur J Nutr 50: 261-269, 2011.

23. Terciolo C, Dobric A, Ouaissi M, Siret C, Breuzard G, Silvy F, Marchiori B, Germain S, Bonier R, Hama A, et al: Saccharomyces boulardii CNCM I-745 Restores intestinal Barrier Integrity by Regulation of E-cadherin Recycling. J Crohn's Colitis 11: 999-1010, 2017.

24. Glover LE and Colgan SP: Hypoxia and metabolic factors that influence inflammatory bowel disease pathogenesis. Gastroenterology 140: 1748-1755, 2011.

25. Flück K and Fandrey J: Oxygen sensing in intestinal mucosal inflammation. Pflugers Arch 468: 77-84, 2016.

26. Colgan SP and Taylor CT: Hypoxia: An alarm signal during intestinal inflammation. Nat Rev Gastroenterol Hepatol 7: 281-287, 2010.

27. Joseph JP, Harishankar MK, Pillai AA and Devi A: Hypoxia induced EMT: A review on the mechanism of tumor progression and metastasis in OSCC. Oral Oncol 80: 23-32, 2018.

28. Scharl M, Weber A, Fürst A, Farkas S, Jehle E, Pesch T, Kellermeier S, Fried M and Rogler G: Potential role for SNAIL family transcription factors in the etiology of Crohn's disease-associated fistulae. Inflamm Bowel Dis 17: 1907-1916, 2011.

29. Yang SW, Zhang ZG, Hao YX, Zhao YL, Qian F, Shi Y, Li PA, Liu CY and Yu PW: HIF-1 $\alpha$ induces the epithelial-mesenchymal transition in gastric cancer stem cells through the Snail pathway. Oncotarget 8: 9535-9545, 2017.

30. Chen S, Chen JZ, Zhang JQ, Chen HX, Yan ML, Huang L, Tian YF, Chen YL and Wang YD: Hypoxia induces TWIST-activated epithelial-mesenchymal transition and proliferation of pancreatic cancer cells in vitro and in nude mice. Cancer Lett 383: 73-84, 2016.

31. Karayiannakis AJ, Syrigos KN, Efstathiou J, Valizadeh A, Noda M, Playford RJ, Kmiot W and Pignatelli M: Expression of catenins and E-cadherin during epithelial restitution in inflammatory bowel disease. J Pathol 185: 413-418, 1998.

32. Mehta S, Nijhuis A, Kumagai T, Lindsay J and Silver A: Defects in the adherens junction complex (E-cadherin/ $\beta$-catenin) in inflammatory bowel disease. Cell Tissue Res 360: 749-760, 2015.

33. Ippolito C, Colucci R, Segnani C, Errede M, Girolamo F, Virgintino D, Dolfi A, Tirotta E, Buccianti P, Di Candio G, et al: Fibrotic and vascular remodelling of colonic wall in patients with active ulcerative colitis. J Crohn's Colitis 10: 1194-1204, 2016. 
34. Andoh A, Fujino S, Okuno T, Fujiyama Y and Bamba T: Intestinal subepithelial myofibroblasts in inflammatory bowel diseases. J Gastroenterol 37 (Suppl 14): S33-S37, 2002.

35. Xue X, Ramakrishnan S, Anderson E, Taylor M, Zimmermann EM, Spence JR, Huang S, Greenson JK and Shah YM: Endothelial PAS domain protein 1 activates the inflammatory response in the intestinal epithelium to promote colitis in mice. Gastroenterology 145: 831-841, 2013.

36. Tang X, Yang Y, Yuan H, You J, Burkatovskaya M and Amar S: Novel transcriptional regulation of VEGF in inflammatory processes. J Cell Mol Med 17: 386-397, 2013.

37. Chen X, Yang G, Song JH, Xu H, Li D, Goldsmith J, Zeng H, Parsons-Wingerter PA, Reinecker HC and Kelly CP: Probiotic yeast inhibits VEGFR signaling and angiogenesis in intestinal inflammation. PLoS One 8: e64227, 2013.
38. Skuli N, Majmundar AJ, Krock BL, Mesquita RC, Mathew LK, Quinn ZL, Runge A, Liu L, Kim MN, Liang J, et al: Endothelial HIF-2 $\alpha$ regulates murine pathological angiogenesis and revascularization processes. J Clin Invest 122 1427-1443, 2012.

39. Ramakrishnan S, Anand V and Roy S: Vascular endothelial growth factor signaling in hypoxia and inflammation. J Neuroimmune Pharmacol 9: 142-160, 2014.

(i) () () This work is licensed under a Creative Commons Attribution-NonCommercial-NoDerivatives 4.0 International (CC BY-NC-ND 4.0) License. 